\title{
Human behavioral pattern analysis-based anomaly detection system in residential space
}

\author{
Seunghyun $\mathrm{Choi}^{1}$. Changgyun $\mathrm{Kim}^{1}$ - Yong-Shin Kang ${ }^{2} \cdot$ Sekyoung Youm $^{1}$
}

Accepted: 18 January 2021 / Published online: 4 February 2021

(C) The Author(s) 2021

\begin{abstract}
Increasingly, research has analyzed human behavior in various fields. The fourth industrial revolution technology is very useful for analyzing human behavior. From the viewpoint of the residential space monitoring system, the life patterns in human living spaces vary widely, and it is very difficult to find abnormal situations. Therefore, this study proposes a living space-based monitoring system. The system includes the behavioral analysis of monitored subjects using a deep learning methodology, behavioral pattern derivation using the PrefixSpan algorithm, and the anomaly detection technique using sequence alignment. Objectivity was obtained through behavioral recognition using deep learning rather than subjective behavioral recording, and the time to derive a pattern was shortened using the PrefixSpan algorithm among sequential pattern algorithms. The proposed system provides personalized monitoring services by applying the methodology of other fields to human behavior. Thus, the system can be extended using another methodology or fourth industrial revolution technology.
\end{abstract}

Keywords Deep learning · Sequential pattern algorithm - Sequence alignment · Monitoring system $\cdot$ Anomaly detection $\cdot$ Human behavioral analysis

Sekyoung Youm

sekyoungyoum@gmail.com

Seunghyun Choi

woofermass0315@gmail.com

Changgyun Kim

tiockdrbs@naver.com

Yong-Shin Kang

yskang7867@gmail.com

1 Department of Industrial and Systems Engineering, Dongguk University, 82-1, Pil-dong, Chung-gu, Seoul, Korea

2 Advanced Institute of Convergence Technology, Suwon, Korea 


\section{Introduction}

In 2018, Korea became an aging society, as defined by the United Nations, and the number of senior citizens living alone is increasing rapidly. However, a workforce shortage exists to monitor senior citizens living alone and deal with possible risks. Therefore, research has recently been conducted to analyze human behavior and apply it to various fields, such as marketing and medicine [1-3]. Fourth industrial revolution technologies, such as artificial intelligence and the internet of things, contribute to analyzing human behavior [4-6]. Research has been emerging to fuse fourth industrial revolution technology with the monitoring system for the care of the elderly and patients living alone in residential spaces [7].

However, existing studies on human behavioral analysis are mostly fragmentary studies using one or two sensors to recognize a situation. The monitoring system attaches a biological sensor or a wearable device to the monitoring target and observes abnormal data in the generated data or observes the current state of the monitoring target using closed-circuit television (CCTV) [8, 9]. However, as the recent analysis of human behavior and the provision of individual services for each individual are the most efficient, monitoring systems in residential spaces should evolve into customized services rather than the existing uniform services [10].

Analyzing human behavior is required at the healthcare level, not for therapeutic purposes. The demand for personalized services is also increasing $[11,12]$. Personalized models are more efficient than generalized models to provide these services. In terms of monitoring systems, daily life in an individual's living space varies from person to person. Moreover, both the beginning and end of the day are different, and each person has his or her life pattern. Therefore, individual behavioral patterns must be analyzed to establish a monitoring system in a residential space. The monitored subject must be observed for a long time to derive the behavioral pattern. The monitored subject's current behavior must also be recognized in detail. However, in general, research on finding behavioral patterns has collected data by observing subjects for a long time but records the current state within a subjective category. Therefore, the current monitoring target status is difficult to determine objectively, and accurate behavioral patterns are challenging to derive because the current status must be determined within a uniform category.

Assuming that human life is a process, process mining can be a useful technique for deriving human behavioral patterns [13]. Process mining techniques can be used to derive process models from a database of the monitored subjects' daily lives and to detect anomalies by comparing the derived process models with running processes [14-16]. A study was recently conducted to derive a process model and detect anomalies in real time rather than derive a process model from a database recorded for a certain period [17-19]. However, applying process mining techniques in their current state to human behavior is challenging because anomaly detection using a process model is performed in the same situation in 
which the beginning and end of a process are the same. However, because human behavior is different in both the first and last actions recorded and daily life varies greatly, a very complex spaghetti-type process model is derived [20]. Therefore, comparing a complex process model with the current process consumes many resources. Process mining also uses a token replay method that sends a single token to a derived process to detect anomalies, which detects the point at which an abnormality occurs through the collected data for a certain period. This method has the disadvantage that abnormality cannot be detected in real time.

Therefore, this study proposes a human behavioral pattern analysis-based anomaly detection system (HBPAADS) that classifies the current human behavior for residential-space monitoring systems and uses the behavior to derive living patterns to detect abnormalities. The deep learning image methodology is used to implement the monitored behavior classifier, collect and analyze the classified behavior, and derive the multifrequency subsequence pattern to implement the HBPAADS in this study. The abnormality is judged by comparing the derived subsequence pattern with the monitored subject's current behavior.

The strength of the proposed system is that the behavioral classifier using deep learning can analyze the behavior in the human living space in more detail. In addition, the system categorizes the monitored subjects' behaviors observed for a certain period rather than using the existing uniform behavior classification method, satisfying the need for personalized monitoring services. In terms of anomaly detection, the system compares the monitoring target's status collected in real time with the pattern using a multifrequency subsequence, not the life patterns derived from process mining. Therefore, it is possible to detect anomalies even in the current state of a complex type of monitoring subject, not in a process mining technique that compares formalized processes with the same start and end. The proposed system also means that the system can be expanded using methodologies or fourth industrial revolution techniques in other areas by applying the existing methodologies applied to humans in various areas, such as manufacturing and biomaterials, to provide customized monitoring services and automatically detect anomalies.

The structure of this study is as follows. In Sect. 2, the existing research on residential space monitoring systems and anomalies detected in monitoring systems is reviewed. Section 3 describes the proposed HBPAADS architecture, descriptions of each module, the implementation method, and the algorithm. Section 4 derives the pattern to verify the HBPAADS and describes the experimental results concerning the accuracy of the anomaly detection. Finally, Sect. 5 describes the summary of this study, its contributions, and future work.

\section{Related work}

The flow of data beyond the normal data pattern is called an anomaly [21]. The basic concept of anomaly detection is predicting the future state based on the current state and discovering the cases compared with the pattern of prederived data. Common anomaly detection uses critical value settings and the support vector machine, among others $[22,23]$. By applying this methodology, the research was conducted to 
find anomalies by observing ordinary human life in various fields, by deriving patterns and comparing them with the current state [24, 25]. In addition, research has been conducted to detect emergencies by finding normal data patterns in residential space monitoring systems and determining data that deviate from these patterns [26].

\subsection{Anomaly detection in the living space}

Anomaly detection in the monitoring system analyzes human behavior and compares the current monitoring target status in real time with patterns or the critical data value. In the early monitoring system, abnormality detection was performed by collecting data from a biosignal sensor. Jang et al. proposed a system measuring the pulse rate using a wristwatch and a ring-shaped sensor to monitor it remotely [27]. If an abnormality exists in the pulse during remote monitoring, it is determined to be an emergency, and an alarm is given. Lical et al. proposed an anomaly detection method using a change in frequency by attaching a radio frequency identification (RFID) reader to a living space and wearing an RFID tag on a monitoring target [28]. A method detects anomalies when a general frequency and a frequency from this is generated according to the movement of the monitored subject.

Subsequently, studies have been conducted to derive patterns by focusing on the monitoring target's behavior, not on the biosignal data, in methods using the critical value and data classification. Carlos et al. derived the pattern using the movement path of the monitoring subject in the monitoring space [29]. Beacons in the form of bracelets were worn on the monitoring targets, and the location data were collected. The experiment was conducted to collect and derive patterns and test the data for 1 month. Virone derived patterns by focusing on specific activities at specific times of day, on a 24-h basis [30]. A motion sensor was installed in the residential area where the monitoring target was active. The residence time was collected, and a pattern was derived. An experiment was conducted to determine the degree of abnormality by randomly comparing the derived data with the daily data.

\subsection{Anomaly detection in the living space}

In the study analyzing human behavioral patterns in the monitoring system, the monitored person's behavior was recorded manually or through sensors. Then, patterns were derived using process mining and data mining techniques.

Timo et al. collected the values of the acceleration, orientation, and GPS sensors using such equipment as smartphones and smartwatches to represent human behavior as a process model [31]. Using the collected data and the predefined device location, environment, and posture, the state of the current monitoring target was recognized. In addition, a predefined activity was recorded by the monitoring target and was used to derive a process model using a fuzzy model, heuristic miner, and inductive miner with sensor values. Finally, the process of deriving a process model through three use cases was presented. 
Seki proposed a detection method using fuzzy variable representation based on Bayesian networks after constructing a monitoring system using omnidirectional cameras [32]. An experiment was conducted to verify the proposed system, deriving a pattern using the dataset of the older adults' average behavioral time for a week, which was investigated by the Statistics Bureau in the Japanese Ministry of Internal Affairs and Communications in 2006.

Tasi et al. analyzed the behavior after collecting the data from the monitoring target using the acceleration sensor attached to the smartwatch [33]. The day of the week that the action occurred and the duration of the action were collected to determine a pattern, and after modeling the distribution of actions by the day of the week, the association between the contexts was analyzed. Afterward, to verify the proposed system, a pattern was derived using CASAS smart home data, and the accuracy of the prediction for the next action was calculated [34].

Song et al. defined the movement and action for an action and recognized it using a three-axis acceleration sensor [35]. Next, using the proposed framework, the probability of moving from the current action to the next action was calculated to derive the action pattern. In addition, because the proposed framework is based on probability, it is possible to predict the next action in real time by linking a mobile phone with a cloud system with a high computational speed.

\section{System architecture}

This section describes the proposed HBPAADS architecture and the main algorithms and implementation methods of the modules that comprise the architecture.

Data Source

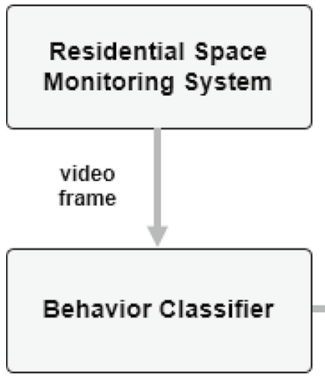

Data Collect
Anomaly Calculation

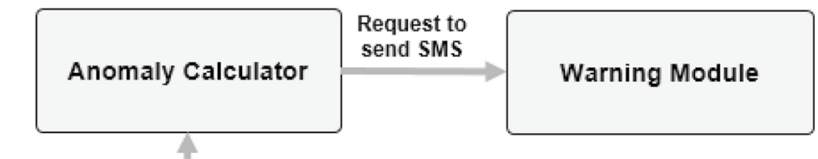

pattern, behavior sequence data set

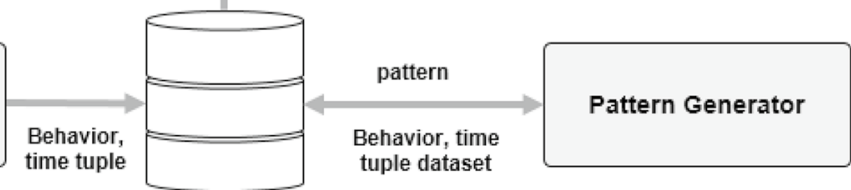

Data Storage
Warning Alarm

Pattern Derivation

Fig. 1 Architecture of the human behavioral pattern analysis-based anomaly detection system 


\subsection{Anomaly detection in the living space}

The HBPAADS consists of five major modules, as illustrated in Fig. 1. The modules are the residential space monitoring system, behavior classifier, pattern generator, anomaly calculator, and warning module.

First, the residential space monitoring system collects image information generated in the residential monitored activity spaces and delivers it to the behavior classifier. A webcam, CCTV, open-source hardware, or another device is used to collect the live video of the monitored target. The behavior classifier analyzes the monitored subject's current behavior from the collected images and stores the data with the time in the database. The pattern generator derives subsequence patterns from the behavioral sequences in the database and stores them. The anomaly calculator is used to detect derived subsequence patterns and abnormal conditions. The last warning module informs the guardian of an abnormal situation through an SMS alarm when the anomaly calculator detects an abnormal condition.

\subsubsection{Residential space monitoring system}

The residential space monitoring system captures the monitored subject's activities in residential and living spaces using a webcam, CCTV, open-source hardware, or another device and delivers the image information to the behavior classifier. Figure 2 displays an example of the residential space monitoring system transmitting a video frame after capturing an image from a camera device.

Using OpenCV and Python's picamera library, each frame is sent to the socket server [36, 37]. The frame sent to the socket server is used to classify the current monitored subject's behavior in the behavior classifier.

\subsubsection{Behavior classifier}

The behavior classifier classifies the current monitoring subject's behavior in the image transmitted from the residential space monitoring system. The behavior classifier uses a behavioral classifier trained using the object detection algorithm that uses the deep learning image methodology. An image of the defined behavior is collected to train the behavior classifier. Images are collected from the web and use the

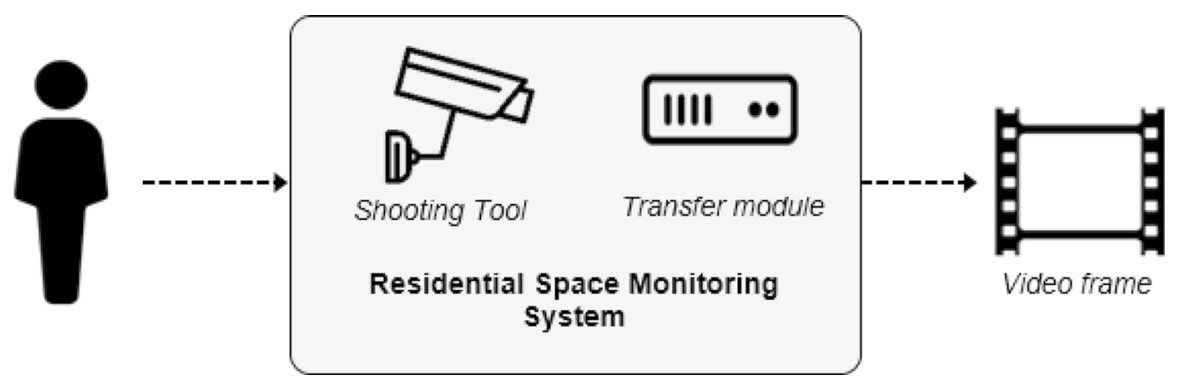

Fig. 2 Example of the residential space monitoring system process 
Fatkun Program, Extreme Picture Finder Program, and so on to download images corresponding to the keywords [38, 39]. The collected images are preprocessed for learning. Image preprocessing includes data labeling to remove unnecessary duplicates of the image, designate the region of interest (ROI) to learn within the image, and create metadata containing the specified ROI and class information.

The deep learning methodology used to train the behavior classifier uses one of the object detection models. The mask regional convolutional neural network (maskCNN), you only look once (YOLO), single-shot detector (SSD), and faster regional CNN (faster R-CNN) are primarily used [40-43]. The above process determines the current monitoring target behavior for each input image frame after learning the behavior classifier. Then, it stores the current monitored behavior in the database with the time. Figure 3 presents the input and output data of the behavior classifier.

The behavior classifier classifies the current monitoring target's behavior through a prelearned behavior classifier using the video frame transmitted from the residential space monitoring system as input data. Afterward, the categorized actions with the time are output in a tuple (time, event).

\subsubsection{Pattern generator}

Collecting monitored behaviors over a period allows the subsequences of monitored behaviors to be derived from the database. After sorting the monitoring target's behavior in the database in chronological order and extracting only the information about the behavior, subsequence patterns are derived. Generally, subsequence patterns can be derived using sequential pattern algorithms and time-series deep learning algorithms.

Recently, a study derived a pattern using the long short-term memory (LSTM) algorithm of the recurrent neural network series, which is a time-series deep learning algorithm [44, 45]. The LSTM algorithm is generally used to predict the next event using the derived pattern. In this study, the LSTM is not used because the degree of abnormality for the entire collected situation is not predicted for the next event. In addition, it takes a very long time to derive a pattern by learning a large volume of data through the LSTM. Therefore, in this study, a pattern was derived using a sequential pattern algorithm. The sequential pattern appears in common data and refers to a multifrequency pattern containing the concept of time [46]. The sequential pattern algorithm is divided into an a priori series represented by

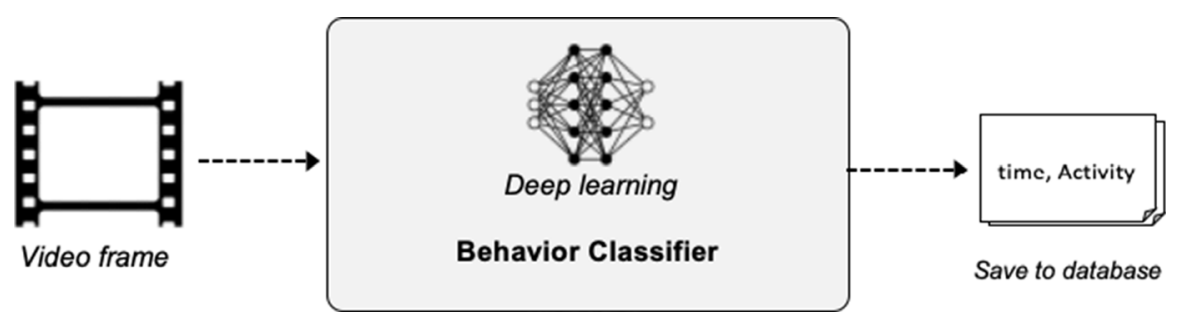

Fig. 3 Example of input and output data for the behavior classifier 


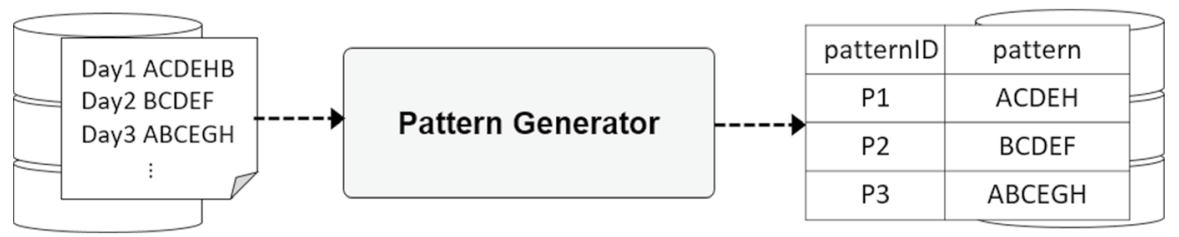

Fig. 4 Example of input and output data for the pattern generator

the generalized sequential pattern and pattern-growth series represented by the PrefixSpan algorithm [47, 48]. Sequential patterns typically use candidate measures to create candidate patterns and derive patterns, but the PrefixSpan algorithm skips the process of creating candidate patterns to improve speed. Therefore, this study derives a subsequence pattern using the PrefixSpan algorithm. Figure 4 depicts an example of the pattern output from the collected behavior sequence.

When the (time, event) dataset from the behavior classifier is delivered, the data are preprocessed to generate a sequence of daily events after sorting them in the order of the time when the events occurred. The preprocessed data are used as input data for the pattern generator. The pattern generator derives patterns using preprocessed data and sequential pattern algorithms, and the derived pattern is output as a pattern ID and subsequence set.

\subsubsection{Anomaly calculator}

The anomaly calculator uses the sequence alignment algorithm to compare the monitored subjects' behavior in the database with the subsequence patterns derived using the pattern generator to determine the degree of abnormality of the current state [49]. The sequence alignment algorithm has been widely used in bioinformatics to analyze the homology of sequences, such as deoxyribonucleic acid (DNA) and ribonucleic acid (RNA). Sequence alignment is largely divided into global alignment, comparing two sequences, and local alignment, comparing which parts of two sequences have high homology. In this study, the local alignment algorithm is used to compare the sequences of different lengths in comparing the behavioral sequences

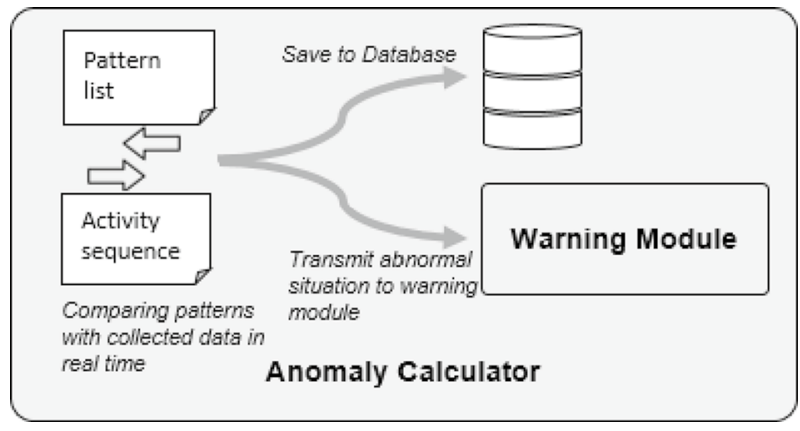

Fig. 5 Example of input and output data for the anomaly generator 
of the monitored subjects stored in the database for a certain period with the subsequence patterns derived during the day. Figure 5 presents an example of determining the degree of abnormality by comparing the derived pattern with the state of the current monitoring target's collected data.

The anomaly calculator calculates the degree of abnormality by comparing the subsequence pattern generated by the pattern generator with the (time, event) dataset delivered from the behavior classifier through the preprocessing process. Unlike the pattern generator, which uses data collected over a long period, the anomaly calculator collects data for about $10 \mathrm{~s}$ to calculate the degree of abnormality over a short period and uses it after preprocessing.

\subsubsection{Anomaly warning module}

The anomaly warning module determines the risk level of the current monitoring target based on the alignment score calculated as a result of the sequence alignment in the anomaly calculator. When a dangerous situation is detected, the anomaly warning module sends an SMS that warns the guardian of the dangerous situation through the internet hosting company's message service.

The alignment score calculated in the sequence alignment used to calculate the degree of the abnormality may vary depending on the length of the data collection. Therefore, after collecting the alignment scores for a certain period, the average score of the alignment is used to set the alignment score that can determine the risk situation.

\subsection{Implementation of HBPAADS}

In this study, to implement the proposed HBPAADS, the system comprises a layer that photographs the monitored subject and a layer that classifies the current monitored subject's behavior and calculates the degree of risk. In this study, we used the open-source hardware Raspberry Pi and a dedicated camera to implement the residential space monitoring system. In addition, we used the object detection algorithm faster R-CNN, the machine running library of Apache Spark, Spark MLlib, and the MySQL database to implement the behavior classifier, pattern generator, anonymous detector, and anomaly warning module and analyzed the current state of the monitored target. Each module implementation uses Python.

\subsubsection{Implementation of residential space monitoring system}

In this study, the open-source hardware Raspberry Pi 3 was used to implement the residential space monitoring system. The camera can be a regular webcam or a dedicated Raspberry Pi camera. In this study, however, a Raspberry Pi camera was used to prevent power shortages when using a typical webcam. We used Python's picamera library to capture the monitoring targets using a Raspberry Pi camera. In general, Raspberry Pi cameras have a very high resolution, which delays the rate at which each frame is sent to the server. Therefore, the camera resolution was adjusted 
for transmission speed. In addition, delays can be prevented using such methods as the protocol definition for wireless transmission [50, 51]. The captured image is transmitted to the socket server for each frame in real time. We use Python's socket library to send data to the socket server, and the internet protocol (IP) of the socket server can be set arbitrarily by the administrator. The behavior classifier classifies the current monitoring target's behavior by analyzing the images transmitted from the residential space monitoring system to each frame's socket server. Then, the system saves the current monitored behavior in the database with the time.

\subsubsection{Implementation of the behavior classifier}

This study trained the behavior classifier using the faster R-CNN algorithm, the object detection algorithm for image deep learning methodology, to construct the behavior classifier. To learn the behavior classifier, it first defined nine behaviors. The defined behaviors are listed in Table 1. The nine defined behaviors were selected as the highest-frequency behaviors through consultation and actual surveys of researchers from the Department of Social Welfare at various universities.

The images were collected using the Extreme Picture Finder System from the web to learn the nine defined behaviors. The Extreme Picture Finder System searches for images related to keywords on the web by entering the keyword for the searched image, and it can be downloaded with a simple click. Duplicate images may exist in image datasets downloaded through the Extreme Picture Finder System, which causes an unnecessary learning process and increases the learning time. Therefore, the VisiPics System is used to eliminate duplicate images [52].

In this study, about 2000 learning datasets for each behavior and about 100 testing datasets were prepared. When the image dataset for the defined behavior was ready, it was labeled. The defined behavior was specified as a class, and the coordinates corresponding to the ROI were extracted from the image from the training dataset and saved as a file in CSV format, which becomes metadata. In this case, the ROI was specified in the form of a rectangle, and the $x$ and $y$ coordinates of each vertex were extracted and stored.

Table 1 Patterns for the behavior classifier

\begin{tabular}{llll}
\hline No & Activity & Substitution & Keyword \\
\hline Activity 1 & Cleaning & A & Clean Room \\
Activity 2 & Drink & B & Drink \\
Activity 3 & Eat & C & Eat \\
Activity 4 & Lying down & D & Lie \\
Activity 5 & Sit & E & Sit \\
Activity 6 & Using a smartphone & F & Using smartphone \\
Activity 7 & Standing & H & Stand \\
Activity 8 & Using a computer & I & Using computer \\
Activity 9 & Watching TV & J & Watching TV \\
\hline
\end{tabular}


Fig. 6 Results of behavior classifier learning

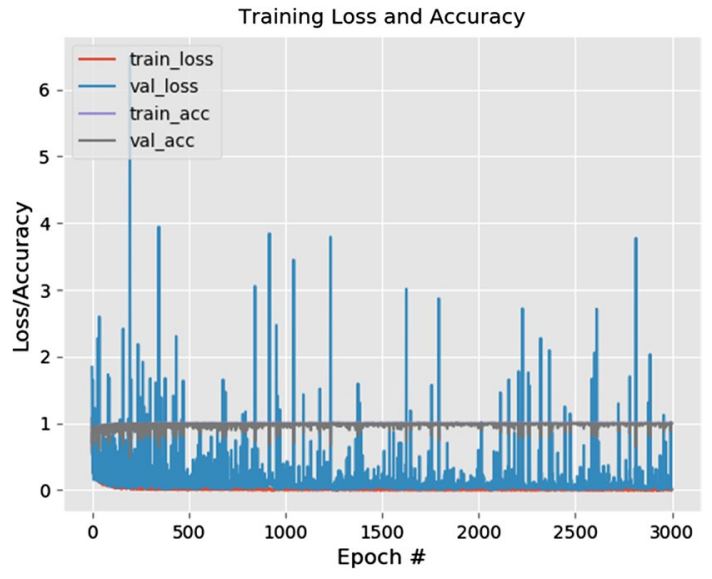

Table 2 Pattern for the behavior classifier

\begin{tabular}{llllll}
\hline & CPU & RAM & HDD & GPU & OS \\
\hline Server & $\begin{array}{c}2.2 \mathrm{GHz}, 8 \\
\text { cores }\end{array}$ & $64 \mathrm{~GB}$ & $512 \mathrm{~GB}$ & RTX2070 & Ubuntu 16.04 \\
\hline
\end{tabular}

A labeling program was used for labeling, and the ROI must be specified manually [53]. Labeling proceeded by directly designating the ROI as a human in a rectangular form. Therefore, no problem occurs when the dataset is small, but the labeling process takes substantial time when the dataset is large. In this case, the recommended method was to locate the shape of a person using an image classifier that had already been learned. Typically, the image classifier displays the searched object in the image as a rectangle. Therefore, using the learned classifier, the person in the image was recognized, and the coordinates of each vertex were extracted for the ROI and displayed as a square. Using this method, even if the dataset increases, labeling can proceed quickly.

After the labeling phase, the faster R-CNN algorithm was trained using the training dataset and metadata. In this study, we set the learning count to 3000 and stored every $500^{\text {th }}$ generated model. As depicted in Fig. 6, the 2500th model with the smallest loss was selected as the behavior classifier. The learning accuracy of the selected model was $91 \%$. The specifications of the computer used for learning are presented in Table 2.

When the behavior classifier was selected, the image captured in real time was analyzed for each frame. When a frame was sent to the socket server, the behavior classifier was used to analyze the current monitored subject's behavior, 


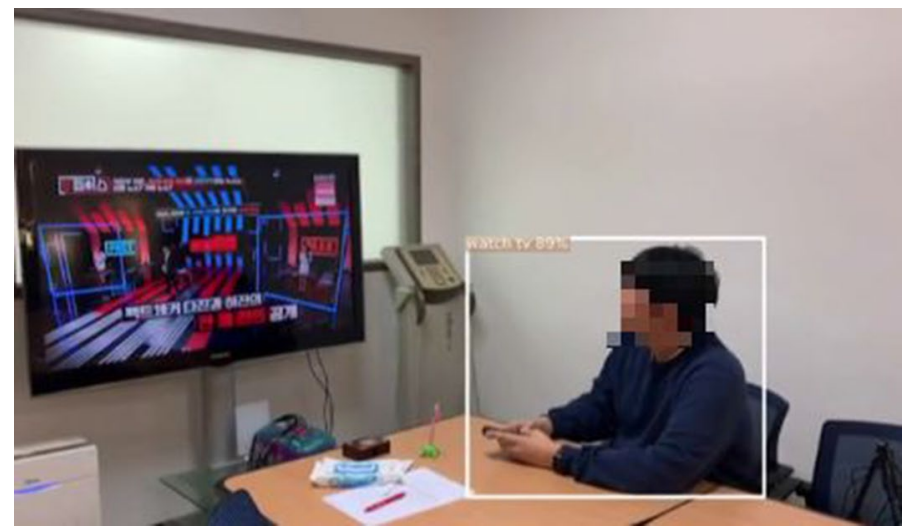

Fig. 7 Results of the behavior classifier

storing it in the database with the time. Figure 7 displays the results of classifying the captured monitoring image and the current monitoring subject's behavior.

\subsubsection{Implementation of the pattern generator}

The pattern generator derives subsequences from the stored behavior over a period. In this study, behavioral sequences stored for one week were used. First, the sequence of actions was listed in chronological order and was cut by the hour. The generated sequences were input into the PrefixSpan algorithm, which is a sequential pattern algorithm, to derive subsequences.

The PrefixSpan algorithm determines the length-1 subsequence pattern of the minsup or greater and creates a projected DB with a prefix. Then, the projected DB is created by deriving the length- 2 subsequence pattern from the projected DB. This process was repeated up to length $N$ to derive the subsequence pattern and store it in the database. In this study, we used Python's MLlib and spark library to implement the pattern generator.

\subsubsection{Implementation of the anomaly calculator}

The anomaly calculator compares the subsequence patterns stored in the database with the behavior sequences of the current monitored subjects accumulated for a certain period. In this study, we use the Smith-Waterman algorithm [54], which is the most used among local alignments, to compare the two sequences. The SmithWaterman algorithm is implemented using Python's biopython library. This study compared the one-minute stored behavior sequence and all derived subsequence patterns in a 1:1 traversal. The comparison result is an alignment score that indicates the abnormality of the current monitoring target. 
Fig. 8 Residential space for the experiment
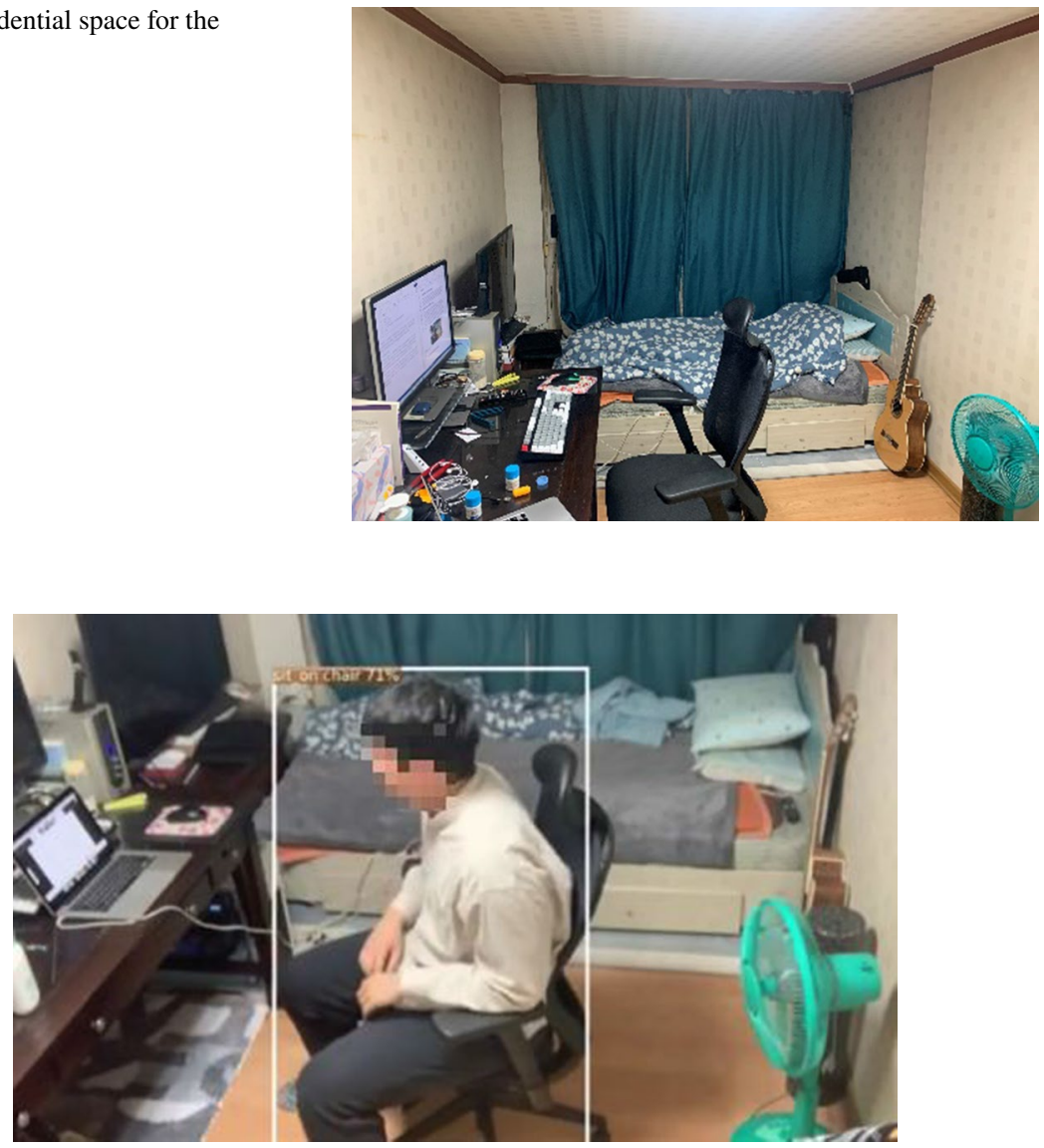

Fig. 9 Results of behavioral detection from real data

\subsubsection{Implementation of the anomaly warning module}

When the anomaly detector's alignment score exceeds a certain value, the anomaly warning module determines that the monitoring target is in a dangerous situation and sends an SMS alarm to the guardian. The SMS transmission uses the message transfer application programming interface (API) of an internet hosting company. In this study, it used Python's requests library to use the message transfer API of an internet hosting company. The ID, password, sender number, recipient number, and risk notification text of the hosting company are sent to the message API using the request post function. 


\section{Experiment of HBPAADS performance}

Data were collected from real residential spaces to verify the HBPAADS proposed in this study. A pattern derivation experiment was conducted using the data. In addition, the sequence alignment execution time was measured according to the realtime data collection period.

\subsection{Applied to actual residential space}

In this study, to verify the proposed system, an environment similar to that of a residential space was built, and data were collected by installing a web camera and a server that can recognize behavior from the monitoring images. Data were collected in a residential space of about $26 \mathrm{~m}^{2}$ for one person, and a camera was installed where the entire residential space was visible. Figure 8 depicts a residential space where some data were collected.

The data were collected for one hour a day for two weeks. Figure 9 presents the analysis of the monitored subject's behavior from the images collected in the experimental environment. The analyzed behavior was saved in the database in text format with the time.

\subsection{Derivation of patterns using real data}

A pattern was derived using the actual collected data to verify the system proposed in this study. Data noise and behavioral false positives were not considered in deriving the pattern. The support of the PrefixSpan algorithm was set to $80 \%$ of the total data. The derived patterns are presented in Table 3.

When the same motion was recognized continuously, the continuous sequence was regarded as one activity to derive a pattern. The PrefixSpan algorithm included in Apache Spark's MLlib library was used to guarantee the accuracy of the derived

Table 3 Derived pattern using the pattern generator

\begin{tabular}{ll}
\hline No & Pattern (top) \\
\hline 1 & D \\
2 & E \\
3 & F \\
4 & I \\
5 & J \\
6 & DF \\
7 & DJ \\
8 & EF \\
9 & EI \\
10 & EJ \\
\hline
\end{tabular}


Fig. 10 Results of the performance test for the anomaly calculator

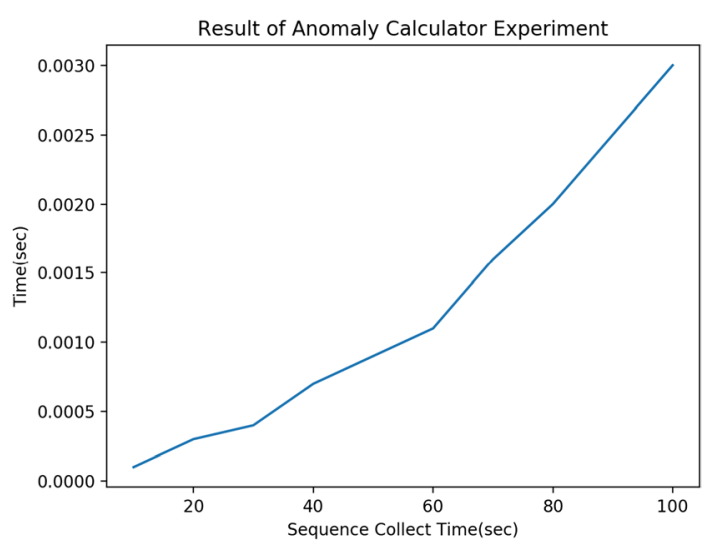

pattern. The derived pattern indicates that most of the subjects watch TV, use a computer, or use a smartphone in a residential space. In addition to the derived pattern, other detected actions were excluded from the pattern because they appeared with a lower frequency than the set value of support. Even if an action is excluded from the pattern, it may be an important action, such as eating or drinking. Therefore, it is very important to set the appropriate support. Moreover, the derived pattern reveals various starting activities. The pattern is derived by assuming that all events of the PrefixSpan algorithm can be starting events. Therefore, the derived pattern can have various starting events resulting from deriving the pattern, starting with events above support.

\subsection{Performance test of the anomaly calculator}

The time taken when the behavior sequence that was collected for $10 \mathrm{~s}$ and all derived patterns were compared 1:1 was measured to verify the performance of the anomaly calculator. In addition, the behavioral sequence was compared with the pattern until it was increased by 10-100 s. A total of 100 experiments were conducted, and all behavioral subsequences for each second were changed.

As presented in Fig. 10, after a certain collection time passes, the execution time of the anomaly calculator rapidly increases. Therefore, when applied to an actual residential environment, the action collection time must be set via experiments.

\section{Conclusion}

In this study, we proposed the architecture of the HBPAADS, which derives and considers the behavioral patterns of the monitoring targets in residential spaces. We verified the system through implementation and experiment. The HBPAADS uses the behavior classifier with the object detection algorithm, a deep learning methodology that can distinguish the monitoring subject's more detailed behavior and 
analyze the current state. In addition, subsequence pattern derivation using the sequential pattern algorithm satisfies the requirements of a customized monitoring system by deriving patterns according to individual life cycles, not behavioral patterns in generalized residential spaces.

Most research on monitoring systems uses data collected from sensors using contact or noncontact sensors. The monitoring target must wear the contact sensor, and if it is not worn, tracking the current monitoring target's state is challenging. For a noncontact sensor, many sensors, such as those on the wall or ceiling of the monitoring space, should be installed to avoid interfering with the monitored subject's daily life. The disadvantage is that the cost of the installation and the sensors is high.

In addition, most studies that analyze human behavior to derive patterns indicate that the monitored subjects themselves record their current behavior. Because this involves the subjective judgment of the monitoring target, the monitoring target's behavior is not objective. In this study, the object detection methodology, a deep learning image method, analyzes the monitoring target's behavior in the monitoring image collected using the camera. This method enables an objective behavioral analysis without attaching specific sensors to the monitoring target or installing them at home.

This study's contribution is that, unlike previous studies, it is possible to provide personalized monitoring services by classifying the monitored subject's current behavior and deriving the monitored subject's life pattern based on the deep learning methodology. In addition, using the deep learning image method, sequential pattern mining, and the sequencing algorithm, various technologies were fused to provide new services.

Because this study is centered on object detection, where the image was used to classify the monitored subject's behavior, a behavior classification method is required that considers more diverse data. The experiment of constructing an experimental environment similar to that of the residential environment in Sect. 4 and collecting actual data reveals that the difference in accuracy according to the shooting angle and distance appeared when the behavior was recognized using only object detection. Therefore, it is necessary to expand the future research and study behavior classification considering biodata and the surrounding data. In addition, data noise was not considered in detecting abnormalities of the monitored subject. In addition, the setting of support has led to cases in which important actions are not included in the pattern. Therefore, research on the optimal support setup is needed.

Acknowledgments The following are the results of a study on the "Leaders in Industry-university Cooperation+" Project, supported by the Ministry of Education and National Research Foundation of Korea.

Open Access This article is licensed under a Creative Commons Attribution 4.0 International License, which permits use, sharing, adaptation, distribution and reproduction in any medium or format, as long as you give appropriate credit to the original author(s) and the source, provide a link to the Creative Commons licence, and indicate if changes were made. The images or other third party material in this article are included in the article's Creative Commons licence, unless indicated otherwise in a credit line to the material. If material is not included in the article's Creative Commons licence and your intended use is not permitted by statutory regulation or exceeds the permitted use, you will need to obtain permission directly from the copyright holder. To view a copy of this licence, visit http://creativecommons.org/licen ses/by/4.0/. 


\section{References}

1. Santacruz KS, Daniel S (2001) Early diagnosis of dementia. Am Fam Phys 64:703-714

2. Eric ES, Marjorie S (2015) Fall detection in homes of older adults using the Microsoft Kinect. IEEE J Biomed Health Inf 19(1):290-301

3. Anthony N, Lee M (2018) An impulse to exploit: the behavioral turn in data-driven marketing. Crit Stud Media Commun 35(2):151-165

4. Ahamad J, Majid AKQ, Abdul SH (2018) Wearable sensor-based human behavior understanding and recognition in daily life for smart environments. In: 2018 International Conference on Frontiers of Information Technology, pp 105-110

5. Muhammad S et al (2020) Human behavior understanding in big multimedia data using CNN based facial expression recognition. Mobile Netw Appl 25:1611-1621

6. Ting Y, Jingen L (2018) Human behavior understanding: From action recognition to complex event detection. In: 2018 ACM Multimedia Conference, 2104-2105

7. Sekyoung Y et al (2019) Development of a methodology to predict and monitor emergency situations of the elderly based on object detection. Multimed Tools Appl 78(5):5427-5444

8. Paradiso R et al (2005) WEALTHY: a wearable healthcare system: New frontier on e-textile. J Telecommun Info Technol 4:105-113

9. We-Duke C et al (2014) Life log big data-based lifestyle analysis and wellness prediction using IoT care service system. J Korean Inst Commun Sci 35(12):17-24

10. Tahan HA, Sminkey PV (2012) Motivational interviewing: building rapport with clients to encourage desirable behavioral and lifestyle changes. Prof Case Manag 17:164-172

11. Yon-Ae A, Han-Jin C (2017) Hospital system model for personalized medical service. J Korea Converg Soc 8(12):77-84

12. Jain KK (2002) Personalized medicine. Curr Opin Mol Ther 4(6):548-558

13. Yang Y, Yu Z, Yukun C, Jianhua F, Xing X (2009) Mining individual life pattern based on location history. In: 2009 10th International Conference on Mobile Data Management: Systems, Services and Middleware, pp 1-10

14. Van Der Aalst W (2011) Process mining discovery, conformance and enhancement of business processes. Springer, Heidelberg

15. van der Wil A, Arya A, van Boudewijn D (2012) Replaying history on process models for conformance checking and performance analysis. Wiley Interdiscip Rev Data Min Knowl Discov 2(2):182-192

16. Ryohei F, Takenhisa Y, Kazuo M (2005) An approach to spacecraft anomaly detection problem using kernel feature space. In: The Eleventh ACM SIGKDD International Conference on Knowledge Discovery and Data Mining, pp 401-410

17. Marwan H, Sergio S, Florian R, Thomas S (2015) Efficient process discovery from event streams using sequential pattern mining. In: Proceedings of IEEE Symposium Series on Computational Intelligence, pp, 1366-1373

18. van Zelst SJ, van Dongen BF, van der Aalst WM (2018) Event stream-based process discovery using abstract representations. Knowl Inf Syst 54(2):407-435

19. Sebastiaan JZ, Alfredo B, Marwan H, van Boudewijn D, van der Wil A (2019) Online conformance checking: relating event streams to process models using prefix-alignments. Int J Data Sci Anal 8:269-284

20. Christian WG, Wil van der A (2007) Fuzzy mining-adaptive process simplification based on multiperspective metrics. In: International Conference on Business Process Management, pp 328-343

21. Varun C, Arindam B, Vipin K (2019) Anomaly detection: a survey. ACM Comput Surv 41(3):15

22. Denning DE (1987) An intrusion-detection model. IEEE Trans Softw Eng 2:222-232

23. Claudio DS, Carlo S, Mario V (2000) To reject or not to reject: that is the question-an answer in case of neural classifiers. IEEE Trans Syst Man Cybern Syst 30(1):84-94

24. Naohiko S et al (2007) Learning motion patterns and anomaly detection by human trajectory analysis. In: 2007 IEEE International Conference on Systems, Man, and Cybernetics, pp 498-503

25. Louis K, Ko N (2009) Anomaly detection in extremely crowded scenes using spatio-temporal motion pattern models. In: 2009 IEEE Conference on Computer Vision and Pattern Recognition, pp 1446-1453

26. Chun Z, Weihua S, Meiqin L (2015) Wearable sensor-based behavioral anomaly detection in smart assisted living systems. IEEE Trans Autom Sci Eng 12(4):1225-1234 
27. In-Hun J, Kwee-Bo S (2007) Ring-type heart rate sensor and monitoring system for sensor network application. J Korean Ins Intell Syst 17(5):619-625

28. Lical $\mathrm{Z}$ et al (2017) Tagcare: using RFIDs to monitor the status of the elderly living alone. IEEE Access 5:11364-11373

29. Carlos $\mathrm{F}$ et al (2013) Process mining for individualized behavior modeling using wireless tracking in nursing homes. Sensors 13(11):15434-15451

30. Gilles V et al (2008) Behavioral patterns of older adults in assisted living. IEEE Trans Inf Technol Biomed 12(3):387-398

31. Sztyler T et al (2015) Discovery of personal processes from labeled sensor data: an application of process mining to personalized health care. In 2015 Proceedings of the International Workshop on Algorithms and Theories for the Analysis of Event Data, pp 31-46

32. Seki H (2009) Fuzzy inference based non-daily behavior pattern detection for elderly people monitoring system. In: 2009 Annual International Conference of the IEEE Engineering in Medicine and Biology Society, pp 6187-6192

33. Tsai M J et al (2016) Context-aware activity prediction using human behavior pattern in real smart home environments. In: International Conference on Automation Science and Engineering, pp168-173

34. Cook DJ (2010) Learning setting-generalized activity models for smart spaces. IEEE Intell Syst 2010(99):1

35. Song J et al (2010) User behavior pattern analysis and prediction based on mobile phone sensors. In: IFIP International Conference on Network and Parallel Computing, pp 177-189

36. Gray B, Adrian K (2008) Learning openCV: computer vision with the openCV library. O'Reilly Media Inc, Newton

37. Jones D (2016) Picamera Documentation. Available: https://picamera.readthedocs.io/en/relea se-1.13/fov. html. Accessed 24 Dec 2019

38. Fatkun. https://chrome.google.com/webstore/detail/fatkun-batch-download-ima/nnjjahlikiabnch cpehcpkdeckfgnohf?hl=ko. Accessed 24 Dec 2019

39. Extreme Picture Finder. https://www.webimagedownloader.com. Accessed 24 Dec 2019

40. Kaiming H, Georgia G, Piotr D, Ross G (2017) Mask R-CNN. In: Proceedings of the IEEE International Conference on Computer Vision, pp 2961-2969

41. Joseph R, Santosh D, Ross G, Ali F (2016) You only look once: Unified, real-time object detection. In: Proceedings of the IEEE Conference on Computer Vision and Pattern Recognition, pp 779-788

42. Wei L et al (2016) SSD: Single shot multibox detector. In: European Conference on Computer Vision, pp 21-37

43. Shaoqing R et al (2017) Faster R-CNN: towards real-time object detection with region proposal networks. Adv Neural Int Process Syst 39(6):1-99

44. Song J et al (2020) Effects of different feature parameters of sEMG on human motion pattern recognition using multilayer perceptrons and LSTM neural networks. Appl Sci 10(10):3358

45. Wang $\mathrm{H}$ et al (2019) Passenger behavior prediction with semantic and multi-pattern LSTM model. IEEE Access 7:157873-157882

46. Rakesh A, Ramakrishnan S (1995) Mining sequential patterns. In: Proceedings of the Eleventh International Conference on Data Engineering, pp 3-14

47. Yu H, Hayato Y (2006) Generalized sequential pattern mining with item intervals. J Comput 1(3):51-60

48. Jian P et al (2001) PrefixSpan: Mining sequential patterns efficiently by prefix-projected pattern growth. In: Proceedings of The 17th International Conference on Data Engineering, pp 215-224

49. David WM (2004) Bioinformatics: Sequence and genome analysis. Cold Spring Harbor Laboratory Press, Cold Spring Harbor

50. Zheng X (2017) The analytics and applications on supporting big data framework in wireless surveillance networks. Int J Soc Humanistic Comput 2(3-4):141-149

51. Jayalakshmi P, Saravanan R (2018) Link stable routing with minimal delay nodes for MANETs. Int J Soc Humanistic Comput 3(1):46-60

52. Visipics. http://www.visipics.info/ Accessed 24 Dec 2019

53. LabelImg. https://github.com/tzutalin/labelImg. Accessed 24 Dec 2019

54. William RP (1991) Searching protein sequence libraries: Comparison of the sensitivity and selectivity of the Smith-Waterman and FASTA algorithms. Genomics 11(3):635-650

Publisher's Note Springer Nature remains neutral with regard to jurisdictional claims in published maps and institutional affiliations. 